4th International Conference Photoinduced Phase Transitions and Cooperative Phenomena, Wrocław 2011

\title{
Quantum and Spontaneous Breakage of Translational Symmetry due to Light Excitation in Photo-Induced Structural Phase Transitions
}

\author{
K. NASU* \\ Institute of Material Structure Science, KEK, 1-1, Oho, Tsukuba, 305-0801, Japan
}

\begin{abstract}
Real time quantum dynamics of the spontaneous translational symmetry breakage due to light excitation in the early stage of photo-induced structural phase transitions is reviewed under the guide of the Toyozawa theory, which is in exact compliance with the conservation law of the total momentum. At the Franck-Condon state, an electronic excitation just created by a visible photon is in a plane wave state, extended all over the crystal. While, after the lattice relaxation having been completed, it is localized as a new excitation. So, is there the shrinkage of the excitation wave function? No! The wave function never shrinks, but only the spatial (or inter lattice-site) quantum coherence (interference) of the excitation disappears, as the lattice relaxation proceeds. This is the breakage of translational symmetry.
\end{abstract}

PACS: $78.90 .+\mathrm{t}$

\section{Adiabatic nature of exciton self-localization and PISPT}

As shown by Toyozawa [1], the photo-induced structural phase transition (PISPT) phenomenon is closely related to the self-localization of an exciton in an insulating crystal. It can be simply described by the following model Hamiltonian $\left(\equiv H_{\mathrm{F}}\right), \hbar=1$,

$$
\begin{aligned}
H_{\mathrm{F}} & =-T_{\mathrm{F}} \sum_{\ell, \ell^{\prime}}\left[F_{\ell}^{\prime+} F_{\ell}+\text { H.c. }\right] \\
& +\sum_{\ell}\left(E_{\mathrm{g}}+6 T_{\mathrm{F}}-\omega_{0} S Q_{\ell}\right) F_{\ell}^{+} F_{\ell} \\
& +\frac{\omega_{0}}{2} \sum_{\ell}\left(-\frac{\partial^{2}}{\partial Q_{\ell}^{2}}+Q_{\ell}^{2}\right) .
\end{aligned}
$$

Here, $T_{\mathrm{F}}(>0)$ is the resonant transfer (energy) of an exciton from a lattice site $\ell$ to its nearest neighbouring sites $\ell^{\prime}$ in a $3 \mathrm{D}$ simple cubic crystal, and $F_{\ell}^{+}$is the creation operator of this exciton at the lattice site $\ell$. It is an intra-atomic (Frenkel, or intra-molecular) exciton, well localized within each lattice site.

As schematically shown in Fig. $1, E_{\mathrm{g}}$ denotes the energy gap of this insulator, while $S$ is the dimensionless coupling constant of this exciton to a site localized phonon, of which energy and dimensionless coordinate are $\omega_{0}$ and $Q_{\ell}$, respectively. The kinetic energy of this phonon will be neglected, because of the adiabatic approximation. In ordinary insulators, $E_{\mathrm{g}}, 6 T_{\mathrm{F}}$ and $\omega_{0} S$ are quantities of the order of $\mathrm{eV}$, while $\omega_{0}$ is $10 \mathrm{meV}$ or so.

The eigenstate $\left(\equiv\left|\Psi\left(Q_{\ell}\right)\right\rangle,\langle\Psi \mid \Psi\rangle=1\right)$ of this $H_{\mathrm{F}}$ will be given as a function of $Q_{\ell}$ and unknown at present, but we determine it under the condition that the total

* e-mail: knasu@post.kek.jp

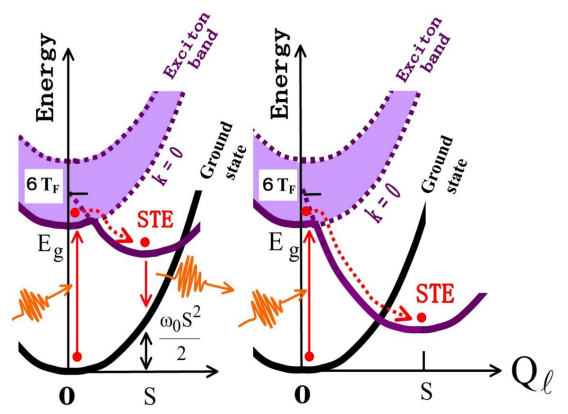

Fig. 1. Schematic nature of exciton self-localization and the start of the PISPT. The luminescent case (left), and the non-luminescent, PISPT case (right). The self-localization formally starts from the exciton band center, whose energy is $6 T_{\mathrm{F}}$ higher than the exciton band edge with the energy $E_{\mathrm{g}}$.

number of the exciton is just one, $\sum_{\ell} F_{\ell}^{+} F_{\ell}=1$. After formally taking the average of $H_{\mathrm{F}}$ with respect to this unknown $|\Psi\rangle$, we can apply the Hellmann-Feynman theorem to Eq. (1), and can get as,

$$
\frac{\partial\left\langle\Psi\left|H_{\mathrm{F}}\right| \Psi\right\rangle}{\partial Q_{\ell}}=0, \quad\left\langle\Psi\left|F_{\ell}^{+} F_{\ell}\right| \Psi\right\rangle=\frac{Q_{\ell}}{S} .
$$

Substituting this Eq. (2) into the original Eq. (1), we also get

$$
\begin{aligned}
& \left\langle H_{\mathrm{F}}\right\rangle=\left(E_{\mathrm{g}}+6 T_{\mathrm{F}}\right)-T_{\mathrm{F}} \sum_{\ell, \ell^{\prime}}\left[\left\langle F_{\ell}^{+} F_{\ell^{\prime}}\right\rangle+\left\langle F_{\ell^{\prime}}^{+} F_{\ell}\right\rangle\right] \\
& -\frac{\omega_{0} S^{2}}{2} \sum_{\ell}\left\langle F_{\ell}^{+} F_{\ell}\right\rangle^{2},
\end{aligned}
$$

where $\Psi$ is omitted in the averages $\langle\ldots\rangle$, for simplicity. We should note that this Eq. (3) holds only at local minimum (or extremum) points in the multidimensional coordinate space spanned by $Q_{\ell}$, since it is obtained by using Eq. (2). 
When the exciton-phonon coupling is sufficiently strong, $6 T<\omega_{0} S^{2} / 2$, according to Shinozuka and Toyozawa [2], we have only two types of minima in the adiabatic potential energy surface of the excited state, as schematically shown in Fig. 1. One is the global minimum with $\left\langle F_{\ell}^{+} F_{\ell}\right\rangle=\delta_{l, 0}$, being localized, say, at the origin $\ell=0$ with a large lattice displacement, $Q_{0}=S$. Its electronic energy $\left(\left(E_{\mathrm{g}}+6 T_{\mathrm{F}}\right)-\omega_{0} S^{2}\right)$, given by the second term of Eq. (1), formally starts from the exciton band center $\left(E_{\mathrm{g}}+6 T_{\mathrm{F}}\right)$, but goes below the exciton band, as a local lattice displacement $Q_{0}$ is self-induced $(0 \rightarrow S)$. It is called self-trapped (or self-localized) exciton (STE) state. The second local minimum is $\left\langle F_{\ell}^{+} F_{\ell}\right\rangle=1 / N$, where $N$ denotes the total number of the lattice sites in the crystal. This is the plane-wave state of the exciton whose wave vector $(\equiv k)$ is zero, $k=0$, and its energy is just the energy gap $E_{\mathrm{g}}$. The final state of the FranckCondon excitation by light is this plane wave state, being the lowest one within the exciton band. While, after the lattice relaxation, as schematically shown by the dashed arrow in Fig. 1, the whole system reaches the STE state. We should also note that, at this largely displaced lattice configuration, even the elastic energy of the ground state, as well as that of the STE, increases up to $\omega_{0} S^{2} / 2$, since the lattice distortion, (the last term of Eq. (1)) is common to all states. If the total energy of this STE state is above the ground state one at this lattice configuration,

$$
\left(E_{\mathrm{g}}+6 T_{\mathrm{F}}\right)-\frac{\omega_{0} S^{2}}{2}>\frac{\omega_{0} S^{2}}{2},
$$

this STE state finally disappears with a luminescence, whose energy is a little smaller that the exciting one, as shown in the left part of Fig. 1. This is the ordinary situation widely realized in the luminescent insulators.

As shown in the right part of Fig. 1, however, if the exciton-phonon coupling is so large as to relax down even lower than the ground state at this largely displaced lattice configuration,

$$
0<\left(E_{\mathrm{g}}+6 T_{\mathrm{F}}\right)-\frac{\omega_{0} S^{2}}{2}<\frac{\omega_{0} S^{2}}{2},
$$

the system becomes non-luminescent, and the STE remains forever within the adiabatic approximation at absolute zero temperature. This is nothing but the start of the PISPT [1].

\section{Dynamics of self-localization}

Let us now see the non-adiabatic quantum dynamics of self-localization, including the kinetic energy term of the phonon in Eq. (1). The wavelength of visible light is quite longer than the lattice constant of the crystal. This means that the wave vector of the visible photon is almost zero, because it is extremely smaller than the wave vectors of an exciton in the first Brillouin zone of this crystal. Consequently, the initial Franck-Condon type excited state $(\equiv|\mathrm{FC}\rangle)$ is the Bloch wave whose total wave vector $(\equiv k)$ is almost zero, having the same translational symmetry as that of the crystal. It is given by

$$
|\mathrm{FC}\rangle=N^{-\frac{1}{2}} \sum_{\ell} \mathrm{e}^{-\mathrm{i} k \cdot l} F_{\ell}^{+}|0\rangle,
$$

$|0\rangle \equiv$ exciton-phonon vacuum. Thus, the probability density of the exciton at each lattice site of the crystal is inversely proportional to $N$ (volume of the crystal),

$$
\left\langle\mathrm{FC}\left|F_{\ell}^{+} F_{\ell}\right| \mathrm{FC}\right\rangle=1 / N,
$$

being normalized to be one within the whole crystal.

Meanwhile, the self-localization mentioned above, is often misunderstood to be a sudden shrinkage of the excitation energy or the excitation wave function from the infinitely extended Bloch state $|\mathrm{FC}\rangle$ to a localized one within a lattice site, say, localized one at the origin of the crystal. This picture of sudden shrinkage, however, is completely wrong. Before, during and even after the self-localization, the wave function never shrinks, as shown by Cho and Toyozawa [3].

They have proposed the following simple but Bloch type self-localized state $(\equiv|\mathrm{STE}\rangle)$,

$$
|\mathrm{STE}\rangle=N^{-\frac{1}{2}} \sum_{\ell} \mathrm{e}^{-\mathrm{i} k \cdot \ell-S\left(F_{\ell}^{+} F_{\ell}\right) \frac{\partial}{\partial Q_{\ell}}} F_{\ell}^{+}|0\rangle, \quad k \rightarrow 0 .
$$

In this Bloch type STE state, the self-localization occurs everywhere with an equal probability. Its probability density at each lattice site of the crystal is unchanged from Eq. (7), and is still inversely proportional to $N$,

$$
\left\langle\mathrm{STE}\left|F_{\ell}^{+} F_{\ell}\right| \mathrm{STE}\right\rangle=1 / N \text {. }
$$

However, as described in Eq. (8), each self-localized state at lattice site $\ell$ induces a large $(S \gg 1)$ lattice distortion only in this site by the following displacement operator for phonons:

$$
\mathrm{e}^{-S\left(F_{\ell}^{+} F_{\ell}\right) \frac{\partial}{\partial Q_{\ell}}} \text {. }
$$

This phonon displacement will appear and disappear according to the presence or the absence of exciton, since it is just proportional to $F_{\ell}^{+} F_{\ell}$. In other words, once this large local lattice distortion occurs, the exciton has heavily dressed in phonons. Hence, even if it tries to move only to a neighboring lattice site from its original one, it has to annihilate all these phonons (larger distortion) and has to make them again at the neighboring site, newly.

This probability can be estimated by the inter lattice-site coherence $(\equiv C(\Delta), \Delta \neq 0)$ of exciton, which is given as,

$$
C(\Delta)=\sum_{\ell}\left\langle\mathrm{STE}\left|F_{\ell^{+}}^{+} F_{\ell}\right| \mathrm{STE}\right\rangle .
$$

It becomes almost zero when the exciton-photon coupling is very strong

$$
C(\Delta) \rightarrow 0\left(=\left\langle 0\left|\mathrm{e}^{-S \frac{\partial}{\partial Q_{\ell+\Delta}}}\right| 0\right\rangle\left\langle 0\left|\mathrm{e}^{-S \frac{\partial}{\partial Q_{\ell}}}\right| 0\right\rangle, S \gg 1\right) .
$$

While, at the initial Franck-Condon state, this inter lattice-site coherence $\left(\equiv C_{\mathrm{FC}}(\Delta), \Delta \neq 0\right)$ is given as 


$$
C_{\mathrm{FC}}(\Delta) \equiv \sum_{\ell}\left\langle\mathrm{FC}\left|F_{\ell^{\prime}+\Delta}+F_{\ell}\right| \mathrm{FC}\right\rangle,
$$

and it remains finite, $C_{\mathrm{FC}}(\Delta)=1$. Thus, we can say, the spatial, or the inter-site quantum coherence of exciton becomes zero when the exciton-phonon coupling is very strong, although it was finite at the initial FranckCondon state $|\mathrm{FC}\rangle$.

This is nothing but the spontaneous translational symmetry breaking, and finally makes a classical and local picture for exciton valid. This relaxation with the large lattice distortion from the Bloch wave to the self-localized one can occur spontaneously even at absolute zero temperature.

\section{References}

[1] Y. Toyozawa, J. Phys. Soc. Jpn. 58, 2626 (1989).

[2] Y. Shinozuka, Y. Toyozawa, J. Phys. Soc. Jpn. 46, 505 (1979).

[3] K. Cho, Y. Toyozawa, J. Phys. Soc. Jpn. 30, 1555 (1971). 